\title{
Caracterização química e mineralógica da incrustação em rede de ferro fundido e potencial de recuperação da capacidade hidráulica
}

\author{
Chemical and mineralogical incrustation characterization and potential of \\ hydraulic performance recovery
}

\author{
Rodrigo Braga Moruzzi \\ Doutor em Engenharia Civil pela Universidade de São Paulo (USP). Professor Assistente da Universidade Estadual de São Paulo Júlio de Mesquita Filho \\ (UNESP) - Rio Claro (SP), Brasil.
}

Fabiano Tomazini da Conceição

Doutor em Geologia Regional pela UNESP. Professor Adjunto da UNESP - Rio Claro (SP), Brasil.

Guillermo Rafael Beltran Navarro

Doutor em Geologia Regional pela UNESP. Professor Assistente da UNESP - Rio Claro (SP), Brasil.

Antenor Zanardo

Doutor em Geologia Regional pela UNESP. Professor Adjunto da UNESP - Rio Claro (SP), Brasil.

\section{Resumo}

O objetivo deste trabalho foi caracterizar química e mineralogicamente os depósitos em rede de ferro fundido e avaliado o potencial de recuperação da capacidade hidráulica em tubulação altamente comprometida pela incrustação. Para tal, foram feitas medidas do coeficiente de resistência (pitometria em trecho isolado de $71 \mathrm{~m}$ ), análises química ( P-ES e ICP-MS), mineralógicas (laminação, difração de raios-X e microscopia eletrônica), dureza (escala de Mohs) e simulações em setor hipotético com cenários distintos (de $\mathrm{C}_{1}$ a $\mathrm{C}_{4}$ ) de troca e substituição da rede. Os resultados indicaram que a rede altamente comprometida tem potencial limitado de recuperação (de 3,9 a 14,0 m $\mathrm{m}^{0,367}$. $\mathrm{s}^{-1}$ ). As caracterizações química e mineralógica indicaram Magnetita e Goethita, com dureza da ordem de 6 na escala Mohs. As simulações demonstraram potências relativas (razões $C_{i} / C_{1}$ ) da ordem de $86,00,1,00$ e 0,02\% em relação ao cenário atual.

Palavras-chave: rede de abastecimento de água; depósitos; incrustações; perda de carga.

\begin{abstract}
The purpose of this work was to characterize chemical and mineralogical the water supply networks and to assess the hydraulic recovery on high-degraded pipe. Thus, the resistance (by pitometric essays), chemical analyses (ICP-MS and ICP-ES), mineralogical analysis (petrographic thin sections, X-ray difractometry and scanning electronic microscopy), hardness (Mohs scale) and simulations for pipe's change and rehabilitations scenarios were investigated (from Scenario 1 to 4). The results showed that the high-degraded pipe had a limited recovery (from 3,9 to $14,0 \mathrm{~m}^{0,367}$. $\mathrm{s}^{-1}$ ). The deposits were formed by oxide and hydroxide of iron (magnetite and goethite), which are materials of high hardness (6 on Mohs scale). The simulations indicated the required power ratio ( $\mathrm{C} / \mathrm{C}_{1}$ ) around 86.00 , 1.00 and $0.02 \%$ in relation to present scenario
\end{abstract}

Keywords: water supply network; deposits; inlay; head-loss.

Endereço para correspondência: Rodrigo Braga Moruzzi - Universidade Estadual Paulista "Júlio de Mesquita Filho" (UNESP) - Departamento de Planejamento Territorial e Geoprocessamento do Instituto de Geociências e Ciências Exatas (DEPLAN/ICGE) - Avenida 24A, 1.515 - 13506-900 - Rio Claro (SP), Brasil - E-mail: rmoruzzi@rc.unesp.br Recebido: 13/02/12 - Aceito: 30/10/12 - Reg. ABES: 226 


\section{Introdução}

A rede de abastecimento de água é, em geral, a parte constituinte mais dispendiosa do sistema de abastecimento de água (SAA), variando de 38 a $76 \%$ do custo do sistema para populações menores que 10 mil ou maiores que 100 mil habitantes, respectivamente (TSUTIYA, 2004). A concepção do SAA depende do porte da cidade e de suas características viárias e topográficas, podendo ser classificada em sistema ramificado, malhado ou misto. Qualquer que seja o tipo de rede, o projeto deve satisfazer algumas condições hidráulicas limitantes, como pressões, velocidades e diâmetros (PORTO, 1998).

As redes constituídas de tubos de ferro fundido $\left(\mathrm{F}^{\circ} \mathrm{F}^{\circ}\right)$, objeto do presente estudo, foram amplamente utilizadas e ainda persistem em grande parte dos sistemas de abastecimento. Apesar de sua resistência mecânica, o envelhecimento e a corrosão favorecem vazamentos nas juntas, arrebentamentos e incrustação. As incrustações ocorrem devido ao depósito de minerais que precipitam na parede do tubo, diminuindo a capacidade de condução hidráulica e causando problemas relacionados à alteração da qualidade da água.

A formação de incrustações na superfície interna da tubulação é determinada por fatores como alcalinidade, dureza, presença de sólidos em suspensão, temperatura, velocidade da água e estado da superfície interna da tubulação. A presença de ferro e manganês na água tratada também favorece a formação de incrustações nas tubulações. A prática tem demonstrado que os tubos de $\mathrm{F}^{\circ} \mathrm{F}^{\circ}$ dúctil não revestidos favorecem o depósito de ferro insolúvel, formando tubérculos, devido à ação de bactérias oxidantes (TSUTIYA, 2004).

A exemplo de muitos municípios brasileiros, a cidade de Rio Claro, localizada no interior do Estado de São Paulo, ainda possui grande parte da rede de abastecimento constituída por tubos de $\mathrm{F}^{\circ} \mathrm{F}^{\circ}$. Estima-se que cerca de $80 \mathrm{~km}$ da rede nesta cidade tenham mais de 50 anos de operação. Em geral, essas linhas foram implantadas no período anterior à década de 1970 e não contavam com revestimento interno. Atualmente, esses condutos estão altamente comprometidos e apresentam incrustações e deposições, evidenciadas diretamente pela análise de amostras de fragmentos da rede, pela alta demanda em reparos e pelo crescente gasto energético, necessário à manutenção das pressões e vazões. Tal situação requereu uma ação urgente do poder público municipal, visando recuperar a capacidade hidráulica e minimizar o gasto energético. Todavia, a troca da rede de abastecimento por tubos novos ultrapassou a capacidade de investimento da prefeitura. Assim, a recuperação da capacidade hidráulica de parte da rede constituiu uma alternativa ao alcance da capacidade de endividamento da municipalidade, por outro lado, é influenciada pelas características químicas e mineralógicas dos depósitos minerais, os quais determinam sua resistência mecânica e a aderência à parede do tubo.

Assim, o presente artigo teve como objetivo investigar a viabilidade da recuperação de tubulações de $\mathrm{F}^{\circ} \mathrm{F}^{\circ}$ de diferentes diâmetros, após manutenção, utilizando técnicas de limpeza e revestimento na rede de abastecimento de $F^{\circ} F^{\circ}$ que constitui parte do SAA da cidade de Rio Claro. Para tal, foi analisada a composição química e mineralógica das incrustações e foram realizados testes de perda de carga na tubulação. A simulação de cenários com diferentes combinações, constituídas por trechos novos, recuperados e nas situações atuais, auxiliou a municipalidade na avaliação da amortização do investimento para cada cenário de limpeza ou troca das tubulações.

\section{Metodologia}

Os ensaios foram divididos em duas fases complementares e indissociáveis. Na primeira, fragmentos de tubulação foram retirados da rede de abastecimento, visando identificar os depósitos e suas características químicas e mineralógicas, bem como a dureza dos seus constituintes, a fim de avaliar a possibilidade de recuperação. Posteriormente, foram realizados ensaios de pitometria em trecho da rede de abastecimento antes e após a operação de sua recuperação, com o objetivo de identificar os valores do coeficiente de resistência (C) da formulação de Hazen-Williams para a rede instalada de $\mathrm{F}^{\circ} \mathrm{F}^{\circ}$. Tais simulações possibilitaram a análise de cenários de perda de energia, os quais foram avaliados comparativamente. A seguir são apresentados os métodos para as duas fases de investigação.

\section{Análise química e mineralógica}

As amostras da tubulação de $\mathrm{F}^{\circ} \mathrm{F}^{\circ}$ com incrustações foram coletadas em diferentes regiões de Rio Claro, onde as tubulações estavam sendo trocadas. Após isso, as amostras foram levadas ao Laboratório de Geoquímica Ambiental, do Instituto de Geociências e Ciências Exatas (IGCE) da Universidade Estadual de São Paulo Júlio de Mesquita Filho (UNESP), Campus Rio Claro, onde foram fotografadas. Na ocasião, as incrustações foram retiradas das paredes dos tubos para obtenção de material para as análises químicas e mineralógicas

Parte de três amostras de diferentes incrustações foi pulverizada, seguindo a rotina convencional de britagem e moagem realizadas no Laboratório de Preparação de Amostras do IGCE/UNESP. Após esta etapa, foram enviadas para as análises químicas realizadas no Laboratório Acme (Analytical Laboratories LTD, Vancouver, Canadá). A concentração dos principais óxi$\operatorname{dos}\left(\mathrm{SiO}_{2}, \mathrm{TiO}_{2}, \mathrm{Al}_{2} \mathrm{O}_{3}, \mathrm{Fe}_{2} \mathrm{O}_{3}, \mathrm{MnO}, \mathrm{MgO}, \mathrm{CaO}, \mathrm{Na}_{2} \mathrm{O}, \mathrm{K}_{2} \mathrm{O}\right.$ e $\left.\mathrm{P}_{2} \mathrm{O}_{5}\right)$ foi obtida por Espectrômetro de Emissão Atômica em Plasma Indutivamente Acoplado (ICP-AES), após fusão utilizando metaborato/tetraborado de lítio e digestão em ácido nítrico diluído, sendo a perda ao fogo (LOI) determinada pela diferença de peso da amostra antes e depois do aquecimento a $1.000^{\circ} \mathrm{C}$ por 4 horas. Os elementos traços (Ba, Sr, Cd, Cu, Ni, Pb e Zn) e terras-raras ( $\mathrm{La}, \mathrm{Ce}, \mathrm{Pr}, \mathrm{Nd}$, Sm, Eu, Gd, Tb, Dy, Ho, Er, Tm, Yb, Lu) foram quantificados em um Espectrômetro de Massa em Plasma Indutivamente Acoplado (ICP-MS), após fusão utilizando metaborato/tetraborado de lítio e digestão por água régia, seguindo os procedimentos internos do referido laboratório.

Outra porção das incrustações foi preparada para a caracterização mineralógica, a qual se iniciou pela imersão de uma pequena parte em resina epóxi. Após a secagem, foi cortada, polida e colada com resina em lâmina 

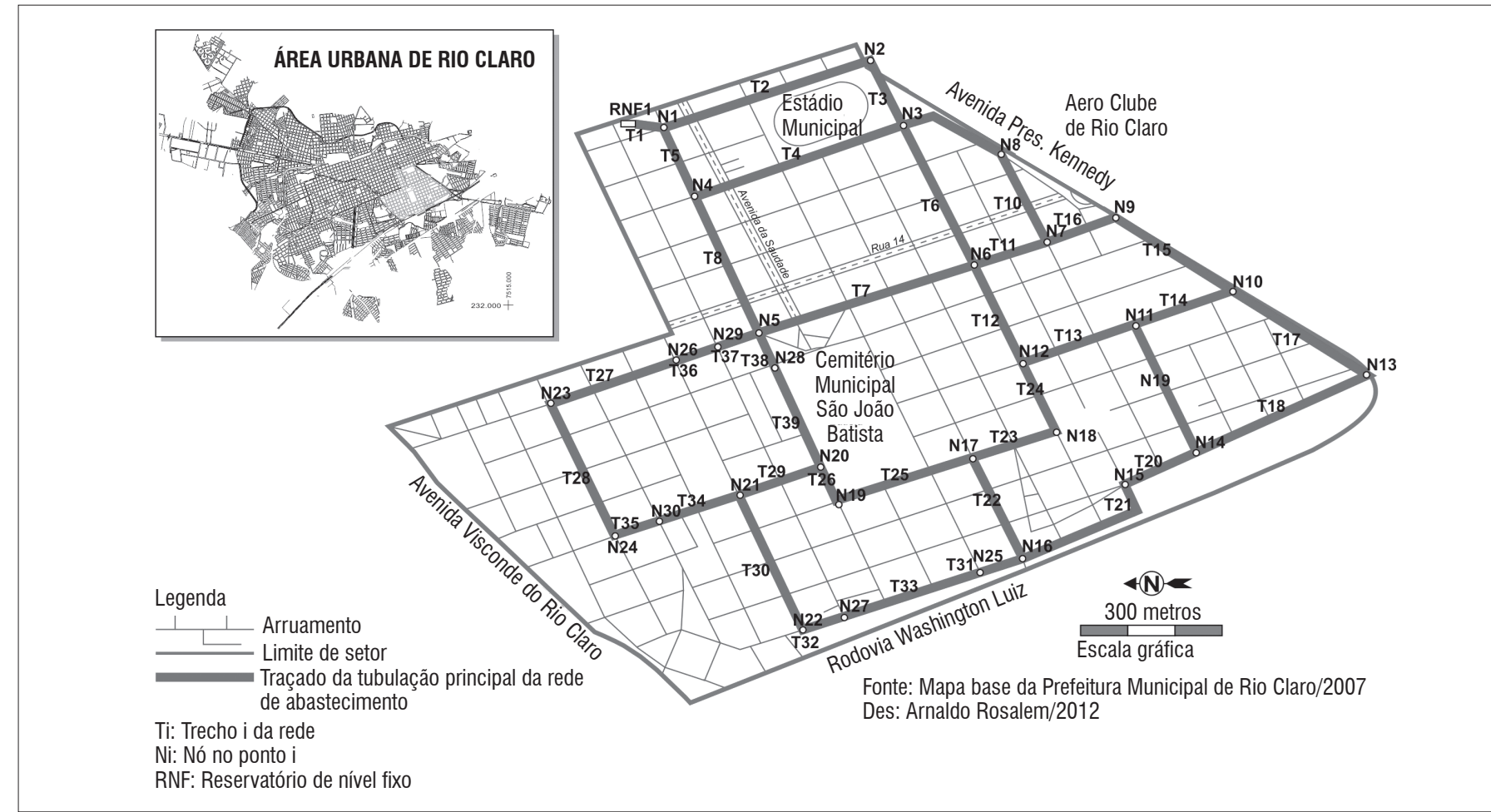

Figura 1 - Setor hipotético de estudo baseado na rede de abastecimento de água do município de Rio Claro (SP). Mapa com traçados das redes fornecido pelo Departamento Autônomo de Água e Esgoto da cidade.

de vidro apropriada para a confecção da seção delgada, no Laboratório de Laminação do IGCE. A seguir, a montagem foi cortada com serra de disco fino, de modo a deixar a amostra com a espessura mais fina possível, submetida a desgaste até chegar a aproximadamente $40 \mu \mathrm{m}$, sendo, a seguir, submetida a polimento com pasta de diamante.

A análise mineralógica das seções delgadas polidas consistiu na observação de microscopia de luz transmitida realizada em microscópio monocular de marca Leitz (Wetzlar SM-Lux Pol), com aumentos de 40, 100, 250 e 630 vezes. As secções delgadas polidas também foram objeto de análise por difração de raios-x em aparelho da Siemens (Diffractometer D5000), com radiação de cobre (tubo de cobre), operando nas seguintes condições: tensão de $40 \mathrm{kV}$ e corrente de $30 \mathrm{~mA}$; uso de filtro de níquel para eliminação de radiação $\mathrm{K} \beta$; faixa de varredura de 3 a $66^{\circ}(2 \theta)$; e passo de $0,02^{\circ}(2 \theta)$, com tempo de acumulação de $1 \mathrm{~s}$ por passo. Ambos os equipamentos estão instalados no Laboratório de Geoquímica do IGCE. Uma seção delgada polida também foi usada para obtenção de microimagens e avaliação da composição mineralógica por meio de microscopia eletrônica de varredura com espectrometria de dispersão de energia (MVE-EDS), marca JEOL, modelo JSM 6330-F, instalada no Laboratório de Nanociências e Nanotecnologia Cesar Lattes do Laboratório Nacional de Luz Sincrotron (LNLS).

\section{Análise hidráulica de cenários de incrustações na rede de abastecimento}

O efeito das incrustações no desempenho da rede de abastecimento de água foi avaliado por meio da análise de desempenho hidráulico, com ênfase nas pressões necessárias para atendimento das vazões de base nos nós da rede. Para tal, foi delineado um setor hipotético baseado na rede de abastecimento de água de Rio Claro (Figura 1). Isso porque a rede do SAA da cidade não é setorizada e o cadastro não está atualizado. Destaca-se que o termo hipotético aplica-se somente à setorização, pois o traçado seguiu conforme a rede existente.

Para a análise de perdas de energia da rede de distribuição foram simulados cenários considerando diferentes condições de incrustações, representadas por também diferentes Cs da equação de Hazen-Williams (Equação 1). Os valores correspondentes ao C foram obtidos por meio de ensaios na rede de abastecimento em um trecho isolado de $71 \mathrm{~m}$ de comprimento para duas situações: antes da intervenção de reabilitação dos trechos (limpeza e revestimento); e após a operação de reabilitação visando à remoção dos depósitos. Foram realizadas duas campanhas de coleta de dados durante 48 horas, após os procedimentos de instalação e calibração dos medidores pirométricos instalados na rede. Para o caso de tubos novos, os valores de $\mathrm{C}$ foram adotados de acordo com as recomendações da literatura especializada (AZEVEDO NETTO, 1998; PORTO, 1998; TSUTIYA, 2004).

Assim, foram elaboradas quatro simulações, cujos cenários diferenciavam-se pelos valores de $\mathrm{C}$ e diâmetros dos trechos. Todas as simulações foram realizadas por meio do software EPANET 2.0, desenvolvido pela United States Environmental Protection Agency (USEPA), e os resultados foram apresentados e discutidos por Curan (2010). O fundamento da simulação hidráulica consistiu na solução numérica das equações de balanço de massa nos nós e de energia nos trechos da rede de abastecimento (Equações 2 a 4). 
Foi empregado o método iterativo híbrido, que efetua a solução por meio de técnicas de minimização numérica com o método de Newton-Raphson. Tal método é incorporado no algoritmo do EPANET ${ }^{\circledR}$, conforme descrito e proposto por Todini e Pilati (1988), seguindo as Equações 5 a 9.

$$
\begin{aligned}
& J=10,65 Q^{1,85} C^{-1,85} D^{-4,87} \\
& \Sigma Q_{i, j}+E_{i}=0
\end{aligned}
$$

$\Delta \mathrm{H}_{\mathrm{i}, \mathrm{j}}=\mathrm{r}_{\mathrm{ij}} \mathrm{Q}_{\mathrm{i}, \mathrm{j}}^{\mathrm{n}}$

\section{Em que:}

$\mathrm{J}=$ perda de carga unitária $\left(\mathrm{m} \cdot \mathrm{m}^{-1}\right)$;

$\mathrm{D}=$ diâmetro interno do tubo $(\mathrm{m})$;

$\mathrm{C}=$ coeficiente de resistência $\left(\mathrm{m}^{0,367} \cdot \mathrm{s}^{-1}\right)$;

$Q=$ vazão no trecho entre os nós $i-j\left(L^{3} \cdot T^{-1}\right)$;

$E=$ vazão concentrada no nó $i\left(\mathrm{~L}^{3} \cdot \mathrm{T}^{-1}\right)$;

$i, j=$ elementos da matriz correspondentes aos nós internos;

$\Delta H=$ perda de carga $(\mathrm{L})$;

$L=$ unidade de comprimento;

$\mathrm{T}=$ unidade de tempo;

$n=$ expoente da vazão;

$r=$ termo de perda de carga, explicitado como:

$$
\begin{gathered}
r=\frac{1}{(0,2785 c)^{1,85}} \frac{L}{D^{4,87}} \\
H_{i}-H_{j}=h_{i j}=r Q_{i j}{ }^{n}+m Q_{i j}{ }^{2}
\end{gathered}
$$

para n=1,85, empregando a equação de Hazen-Williams

\section{Em que:}

$\mathrm{H}$ = cota piezométrica no "nó" i - j (L);

$\mathrm{h}=$ perda de carga total entre os nós i - j (L);

$r=$ termo de perda de carga;

$Q=\operatorname{vazão}\left(\mathrm{L}^{3} \cdot \mathrm{T}^{-1}\right)$;

$m$ = coeficiente de perda de carga localizada.

$$
\sum_{j} Q_{i j}-D_{i}=0 \text { para } i=1, \ldots N
$$

Em que:

$\mathrm{D}_{\mathrm{i}}=$ consumo no "nó" $\mathrm{i}$;

$\mathrm{N}$ = enésimo "nó" da rede.

Neste método, é arbitrada primeiramente uma distribuição de vazões nas tubulações que não tem necessariamente que satisfazer as equações de continuidade nos nós. A primeira aproximação é realizada pelo método linear, servindo de partida para o de Newton-Raphson. Em cada iteração do método, novas cotas piezométricas são obtidas, resolvendo a matriz apresentada na Equação 7.

$A^{*} \mathrm{H}=\mathrm{F}$

Em que:
$A=$ matriz Jacobiana de $(\mathrm{N} \times \mathrm{N})$;
$H=$ vetor de incógnitas em termos de cota piezométrica ( $\mathrm{N}$ x 1);
$F=$ vetor dos termos do lado direito da equação ( $\mathrm{N} x$ 1);
$N=$ nós interiores.

Os elementos da diagonal da matriz Jacobiana e os elementos nulos fora da diagonal são apresentados nas Equações 8 e 9 .

$$
A_{i j}=\sum_{j} p_{i j}
$$

$A_{i j}=-p i j$

Em que:

$p_{\mathrm{ij}}=$ inverso da derivada da perda de carga total no trecho entre os nós i e j em relação à vazão.

Cada termo do lado direito da matriz é composto por uma parcela referente ao balanço de vazão no "nó" à qual é aplicado um fator de correção de vazão. O sistema é resolvido sucessivamente até que a resolução encontrada atenda à precisão determinada.

As seguintes condições e hipóteses simplificadoras foram adotadas para a análise do setor hipotético da rede de abastecimento, conforme descrito em Curan (2010):

- traçado da rede: o sistema simulado neste trabalho considera uma rede principal hipotética, constituída por nove anéis, uma vez que o setor não é, de fato, abastecido somente por um reservatório. A falta de cadastro atualizado e de setorização da rede de abastecimento impossibilitaram a simulação do sistema tal como instalado. Para a definição da rede mestra, baseou-se nos maiores diâmetros e numa rede principal hipotética capaz de suprir todos os pontos de abastecimento do setor;

- densidade populacional: foi considerada a densidade demográfica de saturação para Região Metropolitana de São Paulo, para bairros residenciais populares com lote padrão de $250 \mathrm{~m}^{2}$, já que não há dados oficiais de cidades do interior de São Paulo;

- $\quad$ consumo per capita (l.hab-1 dia $\left.^{-1}\right)$ : foi adotada a média dos valores de consumo per capita de Rio Claro ( $\left.q=156,64 \mathrm{~L}_{\mathrm{hab}} \mathrm{h}^{-1} \cdot \mathrm{dia}^{-1}\right) \mathrm{em} \mathrm{mu-}$ nicípios do interior do Estado de São Paulo de acordo com Tsutiya (2004), concordantes com os valores médios da cidade de Rio Claro;

- $\quad$ vazão específica de distribuição: foram levados em conta os valores do coeficiente de máximos diários $K_{1}$ e horários $K_{2}$ de 1,25 e 1,5, respectivamente, conforme recomendado por Tsutiya (2004). As demandas foram mantidas constantes, sendo o fator de consumo (FC) sempre igual à unidade;

- reservatório: adotou-se reservatório de nível fixo (RNF) cujas propriedades são totalmente independentes do funcionamento da rede. A sua 
localização foi na maior cota do setor, e com entrada de água pelas tubulações de maior diâmetro da rede;

- cotas dos nós: foram determinadas com base nas cotas do terreno e nas recomendações de recobrimento mínimo e profundidades mínima e máxima das valas, de acordo com SABESP (1996);

- cenários: para as quatro simulações foram utilizados os seguintes cenários, os quais foram definidos segundo o planejamento de reabilitação e substituição de Rio Claro, tal como descrito em Curan (2010):

- cenário 1: tubulação no estado pré-reabilitação (10.467,4 m), com valores de diâmetros disponíveis no cadastro do setor disponibilizado pelo Departamento Autônomo de Água e Esgoto (DAAE) de Rio Claro;

- cenário 2: tubulação parcialmente trocada (500 m; 4,78\%) e recuperada $(1.609,4$ m; 15,37\%), com valores de diâmetros disponíveis no cadastro do setor disponibilizado pelo DAAE;

- cenário 3: tubulação totalmente nova (10.467,4 m), com valores de diâmetros disponíveis no cadastro do setor disponibilizado pelo DAAE de Rio Claro;

- cenário 4: tubulação totalmente nova (10.467,4 m) e com diâmetros adaptados às recomendações da antiga norma, Projeto de Norma Brasileira (PNB) 594/77 da Associação Brasileira de Normas Técnicas (ABNT) para as tubulações principais, na qual o diâmetro mínimo deve ser $150 \mathrm{~mm}$ quando abastecer zonas comerciais ou residenciais com densidade igual ou superior a 150 hab.ha-1 $^{-1}$ (TSUTIYA, 2004). A atual norma NBR 12218/1994 da ABNT não recomenda diâmetros mínimos para as tubulações principais, mas que os condutos secundários possuam diâmetro mínimo de $50 \mathrm{~mm}$.

\section{Resultados e discussão}

\section{Composição química e mineralógica das incrustações}

As incrustações apresentam arranjo concêntrico de disposição de coloide, constituindo um arranjo que varia de botroidal a coloforme. As laminações de seção delgada são formadas pela intercalação de magnetita $\left(\mathrm{Fe}_{3} \mathrm{O}_{4}\right)$ e goethita $[(\mathrm{FeO}(\mathrm{OH})]$ (Figura $2 \mathrm{~A})$, constituindo $100 \%$ da composição modal, sendo a porcentagem entre a magnetita e goethita aproximadamente igual. O óxido de ferro também ocorre, formando microglóbulos de óxido de ferro com dimensões de 1 a $5 \mu \mathrm{m}$, que localmente aglomeram-se formando cachos (Figuras 2B e 1C).

Por meio das análises de difração de raios-x, foi confirmada a composição mineralógica das incrustações. Os picos obtidos para diferentes ângulos de incidência são características de estruturas minerais específicas. Os ruídos nos difratogramas podem indicar a presença de substâncias amorfas, ou seja, sem estrutura cristalina característica dos minerais, passíveis de detecção pelo raio-x. Os difratogramas mostram que as incrustações são constituídas predominantemente por magnetita e goethita, com quantidades menores de quartzo $\left(\mathrm{SiO}_{2}\right)$ (Figura 2D). Cabe aqui ressaltar que foi detectada a presença em uma amostra de argilo-mineral denominado alofânio $\left[\left(\mathrm{Al}_{2} \mathrm{O}_{3}\left(\mathrm{SiO}_{2}\right)_{13} \cdot 2,5 \mathrm{H}_{2} \mathrm{O}\right)\right]$. Os resultados obtidos pela petrografia, microscopia eletrônica de varredura e pela
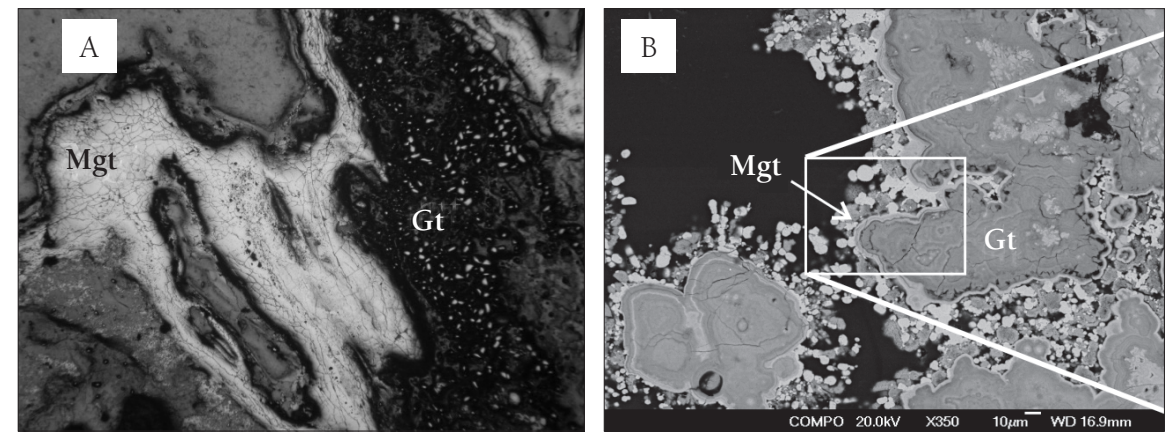

$\mathrm{D}$

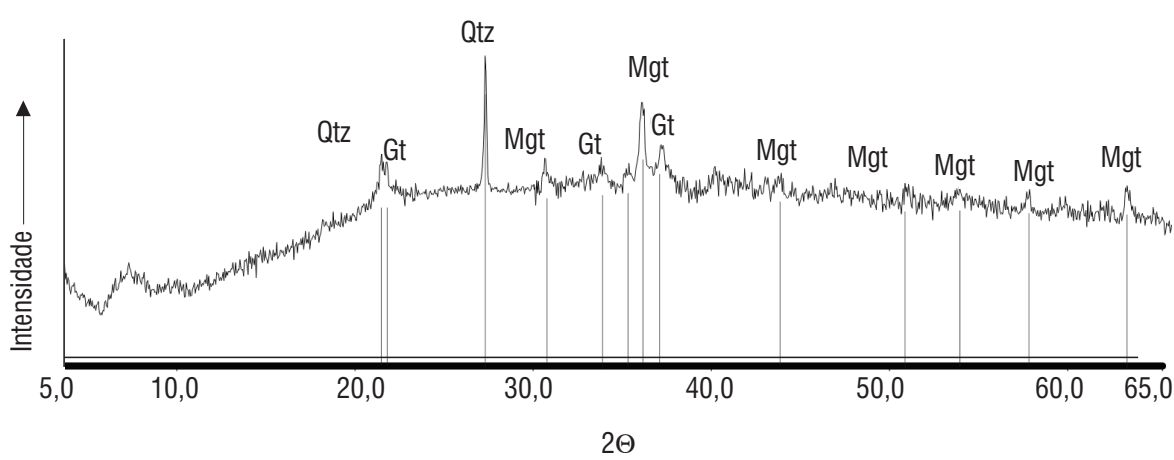

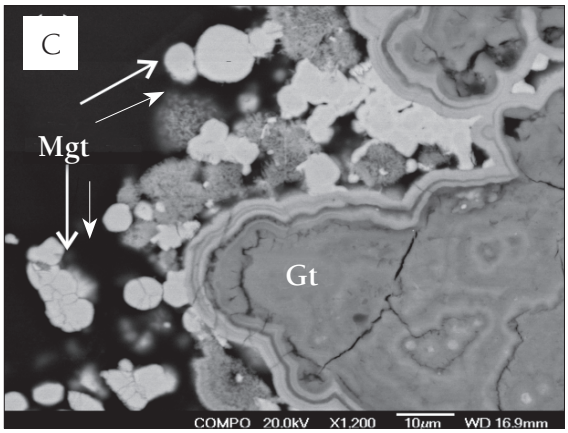

Qtz - Quartzo Mgt - Magnetita Gt - Goethita $2 \Theta$ - ângulo de refração 0 eixo y representa contagem por unidade de tempo

Figura 2 - Fotografias de lâmina de seção delgada polida (A) e microscopia eletrônica de varredura (B e C). Difratograma obtido para a caracterização mineral da incrustação no primeiro ponto coletado na rede de abastecimento de água de Rio Claro (D). 
difratometria de raios-x são semelhantes, mostrando que as incrustações constituem-se principalmente por magnetita e goethita.

Quanto mais fortes as forças de união entre os átomos de um mineral, mais duro ele será, sendo que o grau de dureza está inserido em uma escala que varia entre 1 (talco) e 10 (diamante), conhecida como Escala de Mohs (KLEIN; HURLBUT; DANA, 1993). A composição mineralógica das incrustações tem dureza relativamente alta, ou seja, 5,5 e 6, para a goethita e magnetita, respectivamente. As estruturas minerais presentes caracterizam incrustação de grande resistência mecânica, dificultando a remoção por meio físico quando da grande quantidade de material depositado.

Os resultados referentes à composição química das amostras estão ilustrados na Figura 3. Em relação aos principais óxidos, as amostras apresentam baixas concentrações de $\mathrm{Na}_{2} \mathrm{O}$ de $<0,01$ a $0,11 \%, \mathrm{~K}_{2} \mathrm{O}<0,01 \%$, $\mathrm{TiO}_{2}$ de 0,02 a 0,03\%, $\mathrm{Al}_{2} \mathrm{O}_{3}$ de 0,83 a 1,29\%, MnO de 0,09 a 0,22\%, $\mathrm{MgO}$ de $<0,01$ a 0,08\%, $\mathrm{CaO}$ de 0,01 a 0,14\% e $\mathrm{P}_{2} \mathrm{O}_{5}$ de 0,23 a 0,30\% (Figura 2A). O conteúdo de $\mathrm{SiO}_{2}$ varia de 3,11 a $4,34 \%$ e os valores de $\mathrm{Fe}_{2} \mathrm{O}_{3 \mathrm{~T}}$ e LOI são altos, variando de 80,02 a 80,74\% e 10,0 a 14,3\%, respectivamente. Os resultados das análises químicas para os principais óxidos são condizentes com as análises petrográficas e microscópicas, confirmando a mineralogia observada.

Em relação a elementos traços, as incrustações apresentam teores de $\mathrm{Ba}, \mathrm{Sr}, \mathrm{Cd}, \mathrm{Cu}, \mathrm{Ni}, \mathrm{Pb}$ e Zn, com variações de 40 a 75 mg.kg-1, 3 a 7 mg.kg-1, <0,01 a 5 mg. $\mathrm{kg}^{-1}, 18$ a 28 mg. $\mathrm{kg}^{-1}, 3$ a 25 mg. $\mathrm{kg}^{-1}$ e 22 a $56 \mathrm{mg} \cdot \mathrm{kg}^{-1}$, respectivamente (Figura 3B). A soma do conteúdo total dos elementos terras raras varia de 8,2 a 10,3 mg. $\mathrm{kg}^{-1}$. Os elementos terras raras (ETRs) são os mais úteis de todos os elementos traços e seus estudos têm importantes aplicações em Geoquímica, pois eles têm propriedades físicas e químicas muito similares. Como se pode observar na Figura 3C, as incrustações têm baixos conteúdos de elementos terras raras pesadas (ETRP) em relação aos elementos terras raras leves (ETRL), fato evidenciado pela razão lantânio/lutécio (La/Lu), variando de 145 a 170.

Se os minerais caracterizados neste trabalho fossem originados pela corrosão da própria tubulação, a concentração de ETRs em amostras da tubulação deveria seguir o mesmo padrão encontrado para as incrustações. Contudo, análises químicas da tubulação indicam valores menores que o limite de detecção $\left(<0,05 \mathrm{mg} \cdot \mathrm{kg}^{-1}\right)$ para todos os ETRs. Isso indica que os minerais de ferro encontrados nestes tubérculos podem ser originados pela precipitação do ferro presente na água ou pela influência das bactérias que utilizam o ferro solúvel, tais como Gallionella ferruginea, ou dos gêneros Crenotrix, Leptothrix, Siderocapsa e Sideromonas, que aceleram a oxidação do ferro (GENTIL, 2007; MÜLLER, 2011).

\section{Rugosidade e coeficiente de resistência}

Na Tabela 1 são apresentados os valores de C utilizados nos diferentes cenários. Os valores foram obtidos por meio de ensaios de pitometria e indicam o elevado grau de incrustação presente nas tubulações da rede de abastecimento.
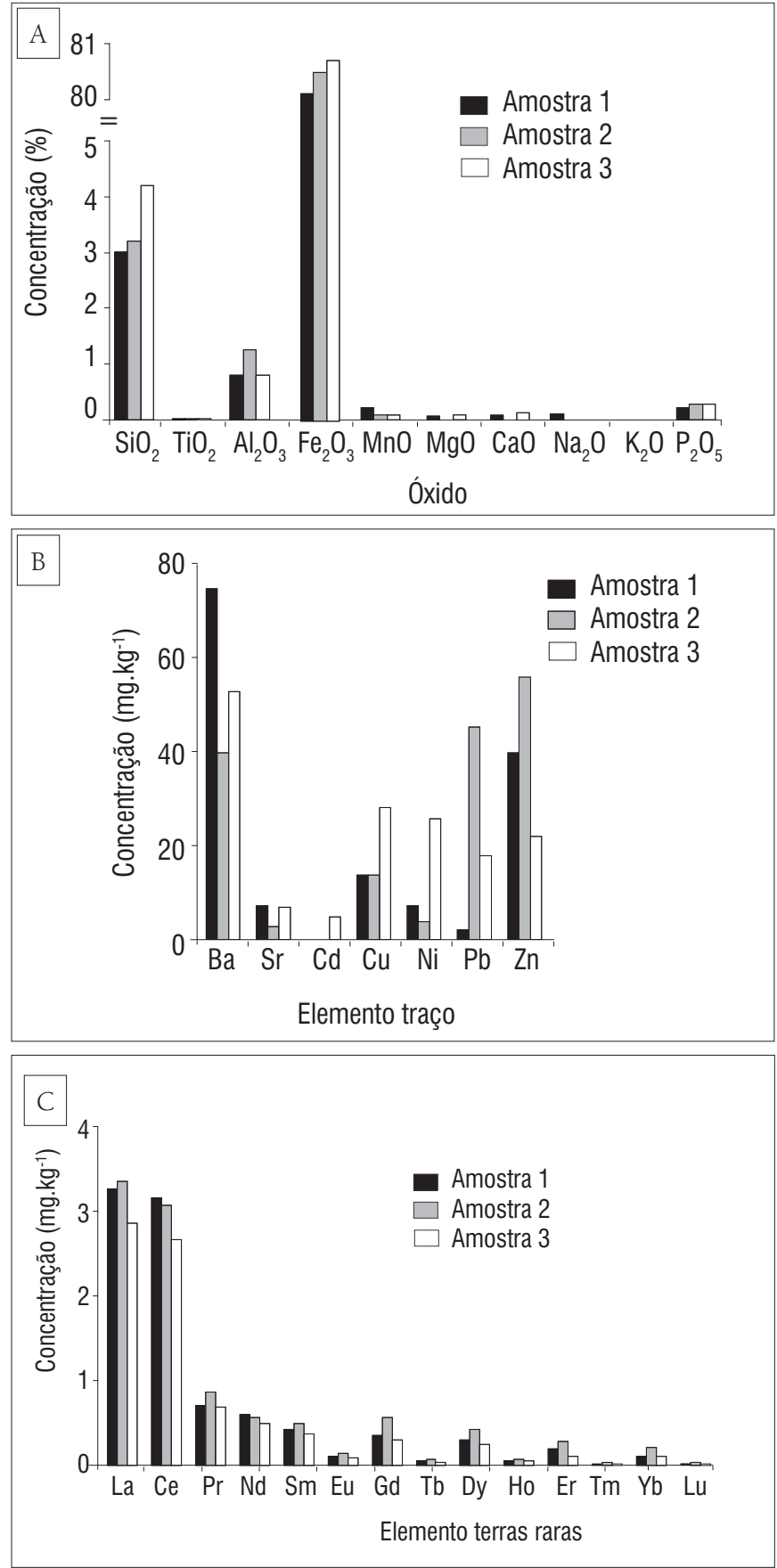

Figura 3 - Resultados obtidos nas análises dos principais óxidos (A), elementos traços (B) e terras raras (C) para as amostras de incrustações retirados do sistema de abastecimento no município de Rio Claro.

A reabilitação da vazão veiculada e da pressão antes e após a operação de limpeza seguida de revestimento pode ser visualizada na Figura 4 para os 289 pontos amostrados. O incremento da capacidade de vazão do trecho isolado da rede após a limpeza e revestimento é evidente, atestando o efeito do processo. A reabilitação do coeficiente em termos relativos foi expressiva, conforme aponta a Figura 5B. Todavia, merece destaque o fato de que o coeficiente de resistência médio em termos absolutos apresenta, mesmo após a limpeza e o revestimento, valores muito aquém dos desejados para uma operação satisfatória da rede de abastecimento (Figura 6). 
Os baixos valores de $\mathrm{C}$ foram atribuídos à alta rugosidade relativa aparente (devido à incrustação) medida em diferentes fragmentos extraídos da rede, cujos valores médios são apresentados na Tabela 2. Considerou-se que a incrustação em alguns fragmentos foi de tal magnitude que causaria praticamente o bloqueio dos trechos da rede, comprometendo sobremaneira a veiculação da água (Figura 7).

Considerando a perda de carga média obtida a partir dos ensaios de pitometria no trecho isolado da rede de $71 \mathrm{~m}\left(\mathrm{~J}\right.$ de $\left.0,03 \mathrm{~m} . \mathrm{m}^{-1}\right)$ e considerando as faixas usuais de C reportados por Porto (1998), correspondentes

Tabela 1 - Valores dos coeficientes de resistência de Hazen-Williams e diâmetros para os distintos cenários.

$\begin{array}{cccc} & \begin{array}{c}\mathrm{C}_{\text {Ferro Fundido }}\left(\mathrm{F}^{\circ} \mathrm{F}^{\circ}\right) \\ \left(\mathrm{m}^{0,367} \cdot \mathrm{s}^{-1}\right)\end{array} & \begin{array}{c}\mathrm{C} \\ \left(\mathrm{m}_{\mathrm{PVC}}^{0,367} \cdot \mathrm{s}^{-1}\right)\end{array} & \begin{array}{c}\mathrm{C}_{\mathrm{PEAD}} \\ \left(\mathrm{m}^{0,367} \cdot \mathrm{s}^{-1}\right)\end{array} \\ \text { Cenário 1 } & 3,87^{\mathrm{a}} & 130^{\mathrm{b}} & - \\ \text { Cenário 2 } & 13,99^{\mathrm{a}} \text { (para tubulação recuperada) } & 130^{\mathrm{b}} & 140^{\mathrm{c}} \\ \text { Cenário 3 } & 3,87^{\mathrm{a}} \text { (para tubulação não trocada) } & 140^{\mathrm{b}} & 140^{\mathrm{c}} \\ \text { Cenário 4 } & - & 140 & 140\end{array}$

C: Coeficiente de resistência de Hazen-Williams.

a Obtidas por ensaios de pitometria em trecho representativo de $71 \mathrm{~m}$ de rede.

SANIT. Universidade Estadual Paulista "Julio de Mesquita Filho" (UNESP). Relatório Preliminar de "Determinação da viabilidade da aplicação do processo de limpeza e revestimento com resina epoxídica pela técnica de turbilhonamento em tubulações de ferro fundido objetivando a reabilitação hidráulica do sistema de distribuição de rede secundária de água potável no município de Rio Claro-SP". Rio Claro.

'Tsutiya (2004).

'JUNDIAÍ. Departamento de Água e Esgoto - DAE. (2010) Sistema de Abastecimento de Água - Normas para elaboração de projeto hidráulico e execução de rede de distribuição de água potável. Disponível em www.daejundiai.com.br/daesite/biblio.nsf/V03.01/normas/\$file/ Pag\%2001 10\%20-\%20NormasRedeAgua.pdf. Acesso em 10 de junho de 2010. à faixa de $\mathrm{C}$ de $90 \mathrm{~m}^{0,367} \cdot \mathrm{s}^{-1}$ para tubo $\mathrm{F}^{\circ} \mathrm{F}^{\circ}$ velho e $\mathrm{C}$ de $130 \mathrm{~m}^{0,367} \cdot \mathrm{s}^{-1}$ para tubo $\mathrm{F}^{\circ} \mathrm{F}^{\circ}$ novo, a vazão veiculada para tubo de $100 \mathrm{~mm}$ seria de 9 a $13 \mathrm{~L} \cdot \mathrm{s}^{-1}$. Os valores de vazão são muito superiores àqueles medidos, mesmo após a reabilitação. Tal fato evidencia a má conservação da rede de abastecimento, a qual apresentou alto grau de incrustação (rugosidade aparente Relativa - $\lambda$ ), e a necessidade de substituição nesses trechos. A idade da tubulação e o longo tempo sem nenhuma operação de manutenção na rede podem ser apontados como causas da impossibilidade de reabilitação adequada do coeficiente de resistência. A presença de ferro na água bruta e na tratada, bem como o uso de coagulantes de sais metálicos à base de ferro empregados na estação de tratamento de água são as prováveis fontes deste metal para a formação de incrustações na rede de abastecimento, conforme corroborados pelas análises químicas apresentadas anteriormente. Este fato sugere a importância de uma investigação em relação à estabilidade química da água tratada, visando minimizar o depósito nas tubulações. Os materiais plásticos usados em tubos, por sua vez, são menos reativos à presença de água quando comparados aos metálicos, sendo o maior problema a interação com solventes orgânicos (CRITTENDEN et al., 2005). Para todos os materiais, os aspectos relacionados à biocorrosão e aos biodepósitos devem ser observados, conforme demonstra estudo de Videla (2002).

A Tabela 2 apresenta os valores médios de diâmetro interno (d), espessura média da incrustação $\left(\varepsilon_{\text {méd }}\right)$ e rugosidade aparente relativa $(\lambda)$ das medidas de fragmentos extraídos da rede de abastecimento.

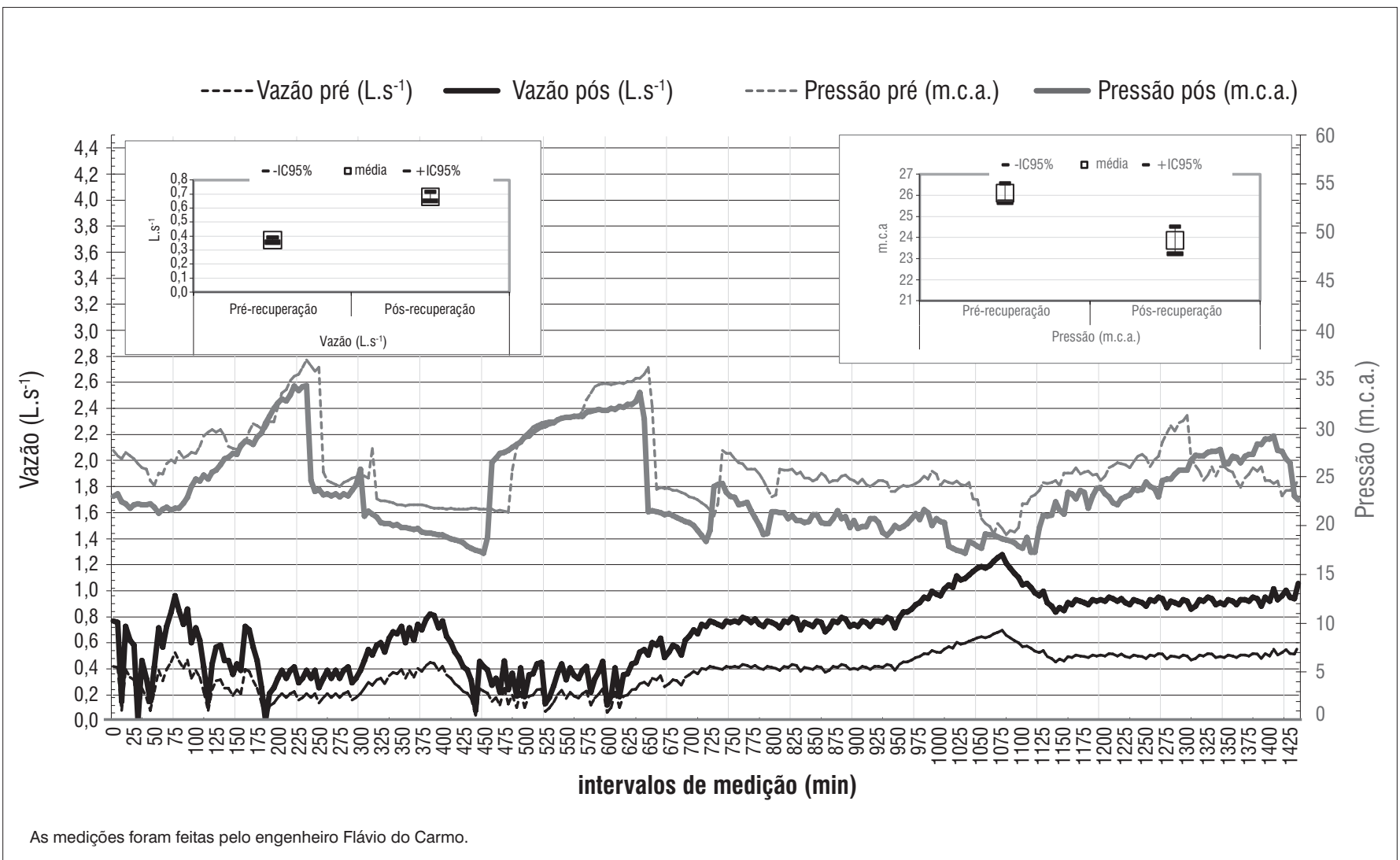

Figura 4 - Vazão veiculada e pressão no trecho submetido aos ensaios antes (nomeados como pré) e após (nomeados como pós) as operações de reabilitação e revestimento. Em detalhe, os resultados para intervalo de 95\% de confiança para a média. Ensaios de pitometria em trecho isolado da rede de ferro fundido de $\mathrm{D} 100 \mathrm{~mm}$ e de $71 \mathrm{~m}$ de comprimento durante campanha de 48 horas. 


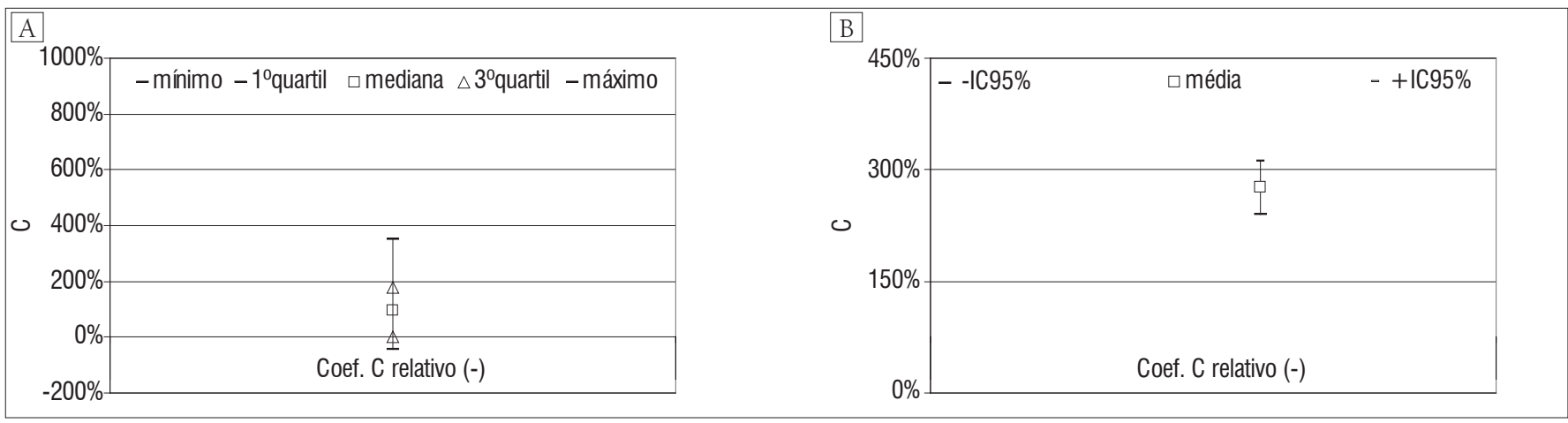

Figura 5 - Reabilitação relativa do coeficiente de Hazen-Williams tendo como referência as condições iniciais (pré-reabilitação). (A) Distribuição dos quartis. (B) Intervalo de Confiança de 95\% (IC95\%) para a média. Ensaios de pitometria em rede de ferro fundido de D100mm em trecho de $71 \mathrm{~m}$ de comprimento durante campanha de 48 horas com 289 pontos amostrados.
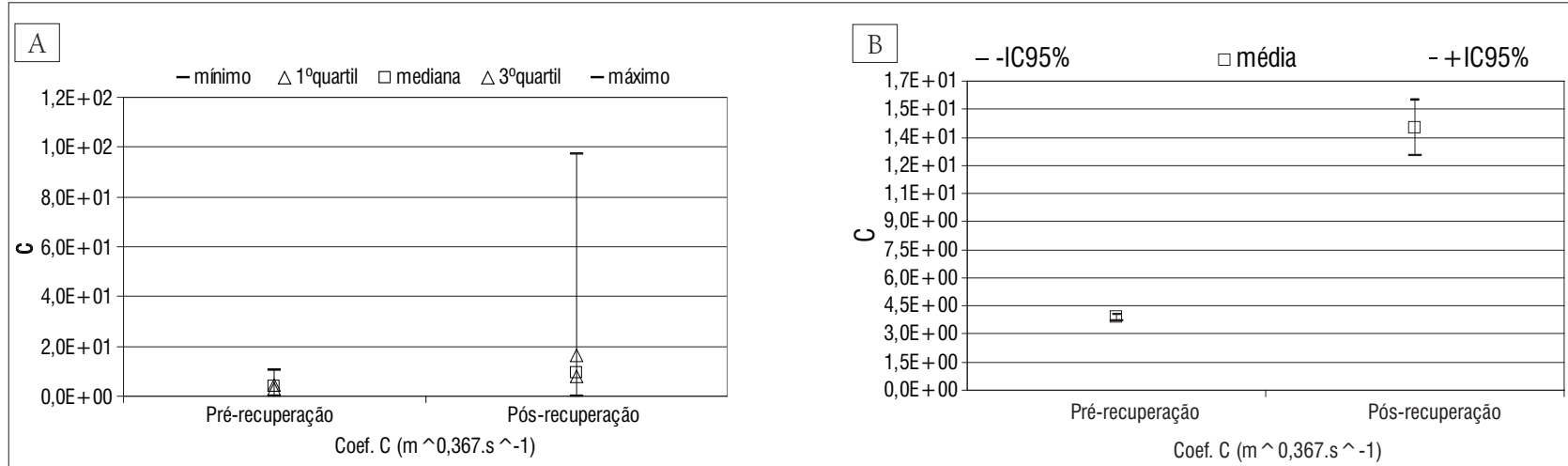

Figura 6 - Variação em termos absolutos do coeficiente de Hazen-Williams. (A) Distribuição dos quartis. (B) Intervalo de Confiança de 95\% (IC95\%) para a média. Ensaios de pitometria em rede de ferro fundido de D100 mm em trecho de $71 \mathrm{~m}$ de comprimento durante campanha de 48 horas com 289 pontos amostrados.

Tabela 2 - Valores correspondentes à espessura média da incrustação medida nas amostras de trechos extraídos da rede de abastecimento da cidade de Rio Claro (SP).

\begin{tabular}{lccc}
$\begin{array}{l}\text { Amostra dos } \\
\text { trechos da } \\
\text { tubulação }\end{array}$ & $\begin{array}{c}\text { Diâmetro } \\
\text { interno do } \\
\text { tubo }(\mathrm{mm})\end{array}$ & $\begin{array}{c}\text { Espessura } \\
\text { média da } \\
\text { incrustação }(\mathrm{mm})\end{array}$ & $\begin{array}{c}\text { Rugosidade } \\
\text { aparente } \\
\text { relativa* }\end{array}$ \\
\hline 1 & 55 & 8,37 & 0,152 \\
\hline 2 & 55 & 10,87 & 0,197 \\
\hline 3 & 50 & 9,12 & 0,182 \\
\hline
\end{tabular}

*O termo "aparente" foi adotado devido à incrustação na parede da tubulação. Os valores indicam rugosidade relativa média em torno de $18 \%$, ocasionando redução média de $66 \%$ da área da seção transversal de escoamento, decorrente da rugosidade média de $0,17 \mathrm{~mm}$ para D50 $\mathrm{mm}$. Nessas condições, a capacidade de veiculação da vazão desejada é seriamente comprometida. O aumento da pressão, necessária para a manutenção da vazão de atendimento pode, mantido o estágio atual de conservação dos tubos, ocasionar sérios problemas para o sistema de abastecimento de água, tais como o aumento das perdas físicas de água tratada e o rompimento de trechos com diferentes classes de pressão. Adaptado de Curan (2010).

\section{Simulação de cenários}

A simulação de cenários foi realizada visando comparar o efeito de diferentes combinações, de acordo com o planejamento de troca e/ou limpeza e reabilitação da rede de abastecimento de Rio Claro,

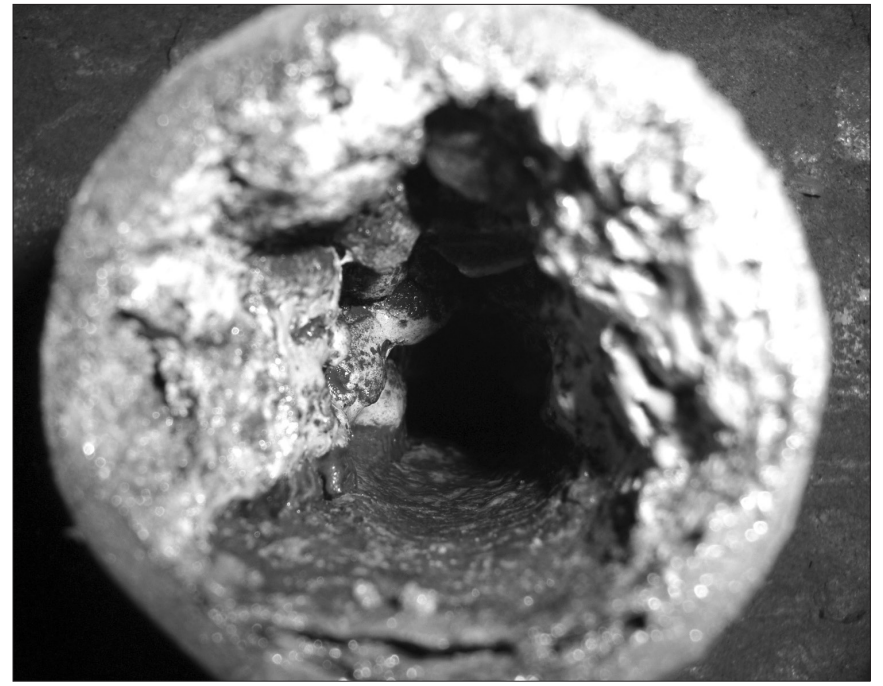

Figura 7 - Fotografia de fragmento de tubo extraído da rede

conforme apresentado por Curan (2010). Dada a inexistência de cadastro atualizado e de setorização dessa rede - o sistema é abastecido por diferentes centrais de reservação interligadas às redes, que, por sua vez, também se comunicam —, optou-se pela definição de uma área de estudo e sua setorização hipotética. Nessa área de estudo, 
foram determinadas e privilegiadas as vazões para atendimento, sendo as pressões resultado das simulações. Assim, os resultados devem ser avaliados em termos comparativos entre os cenários simulados, pois os valores de pressão requeridos para a manutenção das vazões muitas vezes são impraticáveis. Uma exceção se aplica ao cenário 4, no qual a simulação envolveu ainda o redimensionamento do setor hipotético. O setor hipoteticamente delineado atende a 207,8 ha, com consumo estimado de 156,64 L.hab ${ }^{-1}$. dia-1 . A vazão específica de distribuição calculada foi $0,51 \mathrm{~L} \cdot \mathrm{ha}^{-1} \cdot \mathrm{s}^{-1}$.

A Figura 8 apresenta os resultados das simulações dos cenários investigados, contabilizados a partir do nó 5, o qual constitui o primeiro trecho da rede (de montante para jusante em relação à posição do reservatório). Fica claro o efeito da incrustação na perda de energia do sistema evidenciado pelas pressões nos nós quando comparados os quatro cenários investigados. A perda de carga específica é evidentemente maior para os cenários com maiores valores de resistência, representados pelos menores valores de C.
O cenário 1, caracterizado pela ausência de manutenção e substituição de trechos da rede, é o pior em termos de perda de energia. Evidentemente, as vazões demandadas não seriam atendidas, pois as pressões requeridas excedem muitas vezes a máxima recomendada de $500 \mathrm{kPa}$, conforme preconizado pela NBR 12218. No entanto, é importante destacar que a prática na operação do sistema levaria à manutenção de altas cargas de pressão na rede, contribuindo também para o aumento das perdas físicas de água.

A manutenção na rede e a substituição de parte dos trechos por tubos novos, o que é caracterizado pelo cenário 2, amenizam a perda de energia comparativamente ao cenário 1 . Todavia, verifica-se ainda a necessidade da manutenção de altos valores de pressão nos nós para atendimento das vazões requeridas, principalmente nos trechos iniciais da rede.

O cenário 3, que apresenta substituição completa de toda a rede por tubos novos e a manutenção dos diâmetros originais, conta com expressiva melhora, diminuindo e equalizando as pressões na rede. O último cenário analisado (4) versou verificar o efeito da adequação dos diâmetros dos trechos, além da consideração de substituição de toda a rede por tubos novos

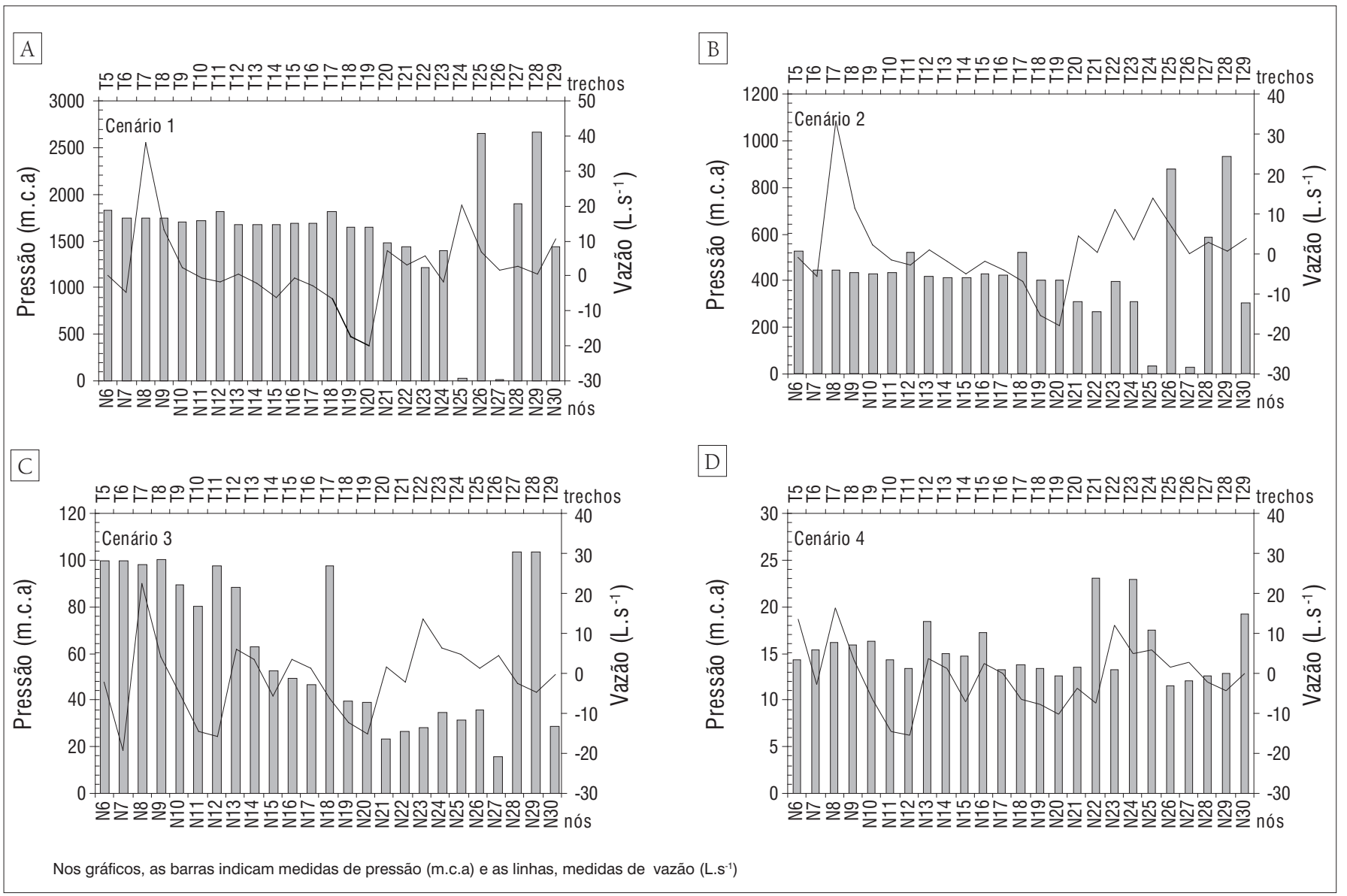

Figura 8 - Resultado das análises hidráulicas dos cenários investigados na rede de abastecimento de água do setor hipotético da cidade de Rio Claro (SP). (A) Cenário 1: tubulação no estado pré-reabilitação (10.467,4 m), com valores de diâmetros disponíveis no cadastro do setor disponibilizado pelo Departamento Autônomo de Água e Esgoto (DAAE) de Rio Claro; (B) Cenário 2: tubulação parcialmente trocada (500 m; 4,78\%) e recuperada (1.609,4 m; 15,37\%), com valores de diâmetros disponíveis no cadastro do setor disponibilizado pelo DAAE de Rio Claro; (C) Cenário 3: tubulação totalmente nova $(10.467,4 \mathrm{~m})$, com valores de diâmetros disponíveis no cadastro do setor disponibilizado pelo DAAE de Rio Claro; (D) Cenário 4: tubulação totalmente nova $(10.467,4 \mathrm{~m})$ e com diâmetros principais mínimos de $150 \mathrm{~mm}$ em zonas comerciais ou residenciais com densidade igual ou superior a 150 hab.ha-1. Adaptado de Curan (2010). 
Tabela 3 - Relação entre as potências necessárias de uma bomba hipotética com rendimento de $67 \%$ para atender à demanda requerida considerando os diferentes cenários investigados, com referência ao Cenário 1.

\begin{tabular}{lcccc} 
Cenário & 1 & 2 & 3 & 4 \\
Relação* & 1 & 0,8667 & 0,0111 & 0,002 \\
\hline
\end{tabular}

*Referente ao cenário 1 .

de PEAD (sem incrustação). Nele, verificam-se valores bem menores de pressão nos nós, além da melhor equalização da rede.

Para facilitar a compreensão do efeito da incrustação no consumo de energia do sistema, as pressões requeridas em cada um dos cenários investigados foram transformadas em potência de bomba hipotética com rendimento de $67 \%$. Tal iniciativa tem valor meramente ilustrativo e não implica na recomendação da instalação desse equipamento eletromecânico em todas as configurações investigadas, no entanto, permite a análise comparativa dos cenários. A Tabela 3 apresenta os valores de potência da bomba hipotética requerida nos diferentes cenários para atendimento da demanda.

Os parametrizando em relação ao cenário 4, verifica-se que o gasto energético decorrente da incrustação é da ordem de 500 vezes maior em relação ao cenário 1 , de 400 vezes em relação ao cenário 2 e de 5 vezes no cenário 3, no qual foram ajustados também os diâmetros dos trechos. Tais resultados deixam evidente a importância da manutenção e troca da rede para o bom funcionamento do sistema de abastecimento de água, minimizando os gastos energéticos e diminuindo os riscos associados à manutenção de elevadas pressões na rede.

\section{Conclusão}

Com base nos resultados apresentados neste artigo, conclui-se que:

- a manutenção da rede de abastecimento deve ser uma prática recorrente, pois a recuperação da capacidade hidráulica é limitada para condutos altamente comprometidos pelas incrustações;

- o valor do C variou de 3,9 a 14,0 $\mathrm{m}^{0,367} \cdot \mathrm{s}^{-1}$, antes e após a reabilitação, respectivamente;

- $\quad$ as caracterizações química e mineralógica indicaram alto conteúdo de ferro na forma de Magnetita e Goethita, com dureza da ordem de 6 na escala Mohs;

- $\quad$ as simulações apresentaram relações de potências hipotéticas requeridas da ordem de 86,00\%, 1,00\% e 0,02\% para os cenários 2, 3 e 4 em relação ao cenário atual, para bomba hidráulica de rendimento de $67 \%$.

\section{Agradecimentos}

Os autores agradecem ao DAAE de Rio Claro; à empresa SANIT, pelo financiamento da pesquisa via FUNEP; ao engenheiro Flávio do Carmo, pelos ensaios de pitometria; e à aluna Roberta Moraes Curan por rodar os cenários no EPANET; além do LME/LNNano/CNPEM pelo suporte técnico durante os trabalhos com microscopia eletrônica de varredura.

\section{Referências}

ASSOCIAÇÃO BRASILEIRA DE NORMAS TÉCNICAS - ABNT. (1994) NBR 12218 - Projeto de rede de distribuição de água para abastecimento público. Rio de Janeiro. ABNT.

AZEVEDO NETTO, J.M. (1998) Manual de hidráulica. 8 ed. São Paulo. Ed. Edgard Blücher, $680 \mathrm{p}$.

COMPANHIA DE SANEAMENTO BÁSICO DO ESTADO DE SÃO PAULO SABESP. (1996) Especificação técnica, regulamentação de preços e critérios de medição. 2 ed. São Paulo. SABESP, 1145 p.

CRITTENDEN, J.C.; TRUSSELL, R.R.; HAND, D.W.; HOWE, K.J.; TCHOBANOGLOUS, G. (2005) Water treatment - principles and design. 2 ed. New York. John Wiley and Sons, 1948 p.

CURAN, R.M. (2010)Caracterização e origem da composição química da incrustação e simulação de cenários de perda de energia em setor da rede de abastecimento de água da cidade de Rio Claro - SP. 65p. Monografia (Graduação em Engenharia Ambiental) - Instituto de Geociências e Ciências Exatas, Universidade Estadual Paulista, Rio Claro.

GENTIL, V. (2007) Corrosão. 5 ed. Rio de Janeiro. LTC, 353 p.

KLEIN, C.; HURLBUT, C.S.; DANA, J.D. (1993) Manual of mineralogy. 21 ed.
New York. Wiley \& Sons, 681 p.

MÜLLER, W.E.G. (2011) Molecular biomineralization - aquatic organisms forming extraordinary materials. Springer, $414 \mathrm{p}$.

PORTO, R.M. (1998) Hidráulica básica. São Carlos. EESC/USP Projeto REENGE, $540 \mathrm{p}$.

RIO CLARO. Departamento Autônomo de Água e Esgoto - DAAE. (2009). Disponível em: mww.daaerioclaro.sp.gov.br. Acesso em 20 de julho de 2009.

SANIT ENGENHARIA. (2009). Disponível em www.sanit.com.br/servicos.asp ?id=Revestimento\&ling=ptg\&st=flash. Acesso em 15 de julho de 2009.

Todini, E; Pilati, S. (1988) A gradient algorithm for the analysis of pipe networks. In: COULBECK, B. \& CHOUN-HOU, O. (EDS) Computer applications in water supply, Vol I - Systems analysis and simulation. Research Studies Press. Taunton, UK, p. 1-20.

TSUTIYA, M.T. (2004) Abastecimento de água. 2 ed. DEHS-EPUSP. São Paulo, $643 \mathrm{p}$

VIDELA, H.A. (2002) Prevention and control of biocorrosion. International Biodeterioration \& Biodegradation, v. 49, n. 4, p. 259-270. 